\title{
Observation of the Electrochemically Active Surface Area in a Proton Exchange Membrane Fuel Cell
}

\author{
Julio Luna, Student Member IEEE, Elio Usai, Member IEEE, Attila Husar, and Maria Serra
}

\begin{abstract}
In this paper a methodology for the estimation of the Electrochemically active Surface Area (ECSA) of a Proton Exchange Membrane Fuel Cell (PEMFC) is developed. Specifically, the ECSA in the Cathode Catalyst Layer (CCL) is estimated, which makes it possible to quantify the amount of active platinum (Pt) in the CCL. In order to estimate the ECSA, the internal conditions of the PEMFC have to be known. A model-based observer for the distributed parameter dynamics is designed. From the observation of the distributed states, the ECSA can be estimated as well. With the aim of representing the most relevant phenomena that affects the PEMFC voltage, the simulation model includes a two-phase water model and the effects of liquid water on the CCL. The results of the methodology are discussed in a simulation environment using the New European Driving Cycle (NEDC) as a case study.
\end{abstract}

\section{INTRODUCTION}

As the necessity to invest in renewable energies increases in modern society, fuel cells are gaining traction as an efficient and clean technology for the production of electrical energy and heat. Within this context Proton Exchange Membrane Fuel Cells (PEMFC), which use hydrogen as fuel, provide high power densities while operating at lower temperatures, which reduces the cost of materials and maintenance. PEMFC are suitable for a broad range of applications, such as stationary, Combined Heat and Power (CHP), portable systems and automotive. Performance in PEMFC-based systems is greatly influenced by the internal conditions of the fuel cell. Specifically, durability and efficiency are related to the concentration of reactants in the Catalyst Layer (CL), the humidification of the membrane and the liquid and vapour of water within the PEMFC [1].

The Electrochemically active Surface Area (ECSA) at the Cathode Catalyst Layter (CCL) is a measure of the total active platinum $(\mathrm{Pt})$ surface [2], giving a numerical value of the effective area where the reaction can occur. The ECSA affects the output voltage of the PEMFC and it depends on the Pt loading of the catalyst, the pore distribution and the state of hydration of the CL. Pore distribution and Pt loading of the catalyst are specifications given by the manufacturer. Moreover, these parameters degrade and fluctuate during the lifetime of the fuel cell. Henceforth, hydration is the only degree of freedom left to control the ECSA in a PEMFC.

J. Luna, A. Husar and M. Serra are with the Institut de Robòtica i Informàtica Industrial, CSIC-UPC. Llorens i Artigs 4-6, 08028 Barcelona, Spain (e-mail: \{jluna,ahusar,maserra\}@iri.upc.edu)

E. Usai is with the Dipartimento di Ingegneria Elettrica ed Elettronica (DIEE), Università di Cagliari, Piazza d'Armi, Cagliari, Italy (e-mail: eusai@diee.unica.it)
PEMFC hydration is affected by different mechanisms: the water generated at the Cathode Catalyst Layer (CCL) which increases with the demanded current load, changes in the humidity of the input reactant fluxes, changes in the PEMFC temperature, internal diffusion from higher to lower water concentration regions and the water electro-osmotic drag caused by the protons movement across the membrane. These mechanisms are highly nonlinear and directly related to the PEMFC efficiency. Monitoring and controlling the state of hydration of the PEMFC is crucial when operating these systems. Obtaining the amount of water concentration at the CCL can be a challenging task from the technological point of view since PEMFCs are hermetic systems.

Utilising the measurements commonly available in PEMFCs, nonlinear observers with disturbance and unknown input reconstruction can be synthesised to estimate the internal PEMFC variables (i.e. reactants and water concentration at the catalyst sites). Moreover, recent studies [3, 4] have concluded that it is possible to efficiently use distributed parameters models in order to implement observers that take into account the spatial variations of the internal variables in PEMFCs.

The main contribution of this paper relies on the estimation of the ECSA along the CCL in the direction of the gas channels based on a nonlinear distributed parameters model [5] of a PEMFC. To achieve this, a High Order Sliding Mode (HOSM) observer [6] is implemented in order to estimate the full profile of all the reactants and water concentrations along the gas channels of the PEMFC. Afterwards an observer for the diffused concentrations is used to estimate the amount of reactants available at the catalyst layer of the PEMFC. Finally, an algebraic equation permits to compute the ECSA from the estimated concentrations at the CCL. In this paper, the estimation of the ECSA is done in a simulation environment. The simulation model is derived from the discretisation of the partial differential equations that describe the nonlinear dynamics of the system, considering spatial discretisations along the gas channels for the gas concentrations. The water model includes an additional discretisation dimension perpendicular to the gas channels to model the pore-filling dynamic effect.

The paper is organised as follows. In Section II, the complete simulation model is introduced. In Section III the observation problem is stated and the observation model is described to design the ECSA observer in Section IV. The case study and simulation results are presented in Section V. Finally, in Section VI the results of this paper are reviewed and some research lines for future work are proposed. 


\section{PROBlem FORMULATION}

\section{A. System description}

The system studied in this paper is a single-channel PEMFC. The PEMFC is simulated to substitute reality adopting a $1+1 \mathrm{D}$ or quasi-two dimensional distributed parameters model [5]. See Figure 1 and [3] for an overview of the PEMFC and the considered coordinate frame. The PEMFC has a channel length $(L)$ of $0.4 \mathrm{~m}$ and a total surface area $A_{\text {geo }}$ of $25 \mathrm{~cm}^{2}$. The PEMFC runs on humidified hydrogen and air. The temperature is considered to be constant.

The simulation model considers the gas channels, Gas Diffusion Layers (GDL), the CLs and the electrolyte membrane of the PEMFC. Moreover, at the CCL a two-phase water model is modelled to better represent the fuel cell voltage dynamics. All modelling variables and parameters are collected in Table I.

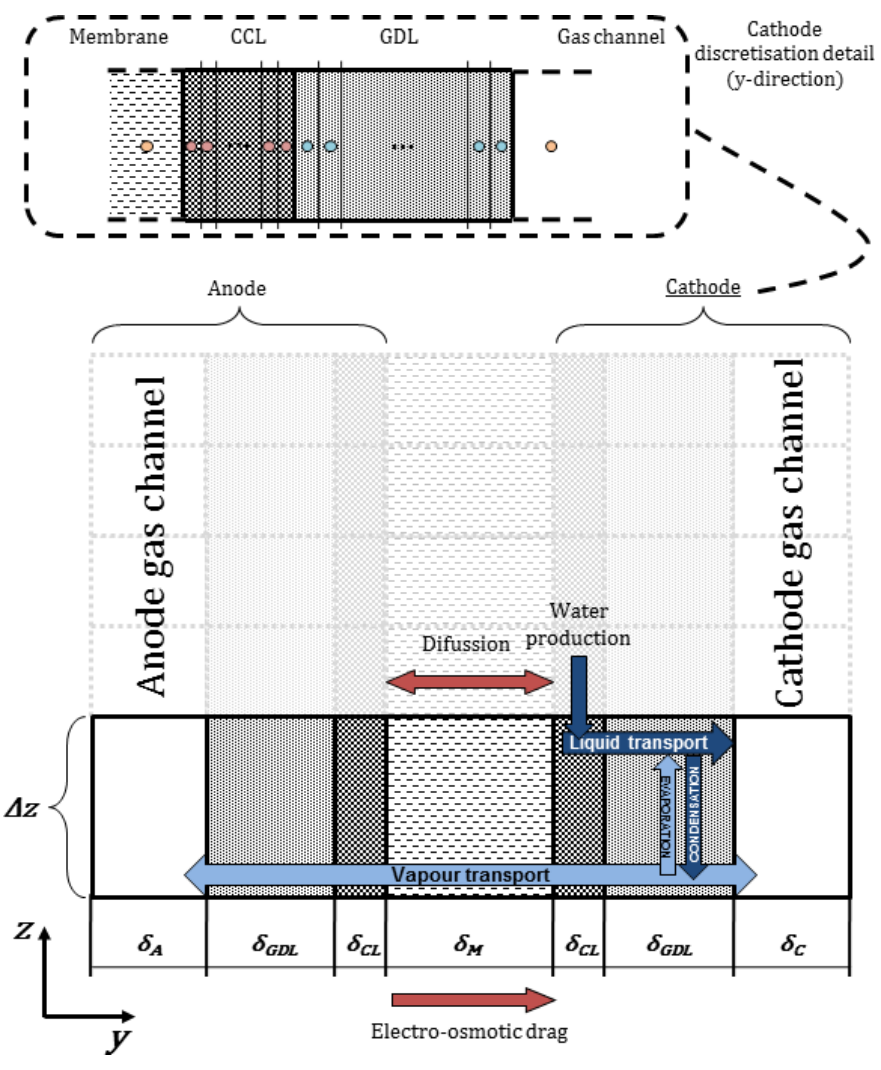

Fig. 1. Discretisation volumes and water transport mechanisms

\section{B. PEMFC model}

1) Electrochemical model: The single-cell voltage is modelled with the Butler-Volmer equation [1] applied to the CCL

$$
V_{f c}=E_{r}-\frac{R T}{\alpha 2 F}\left[\log \left(\frac{i}{i_{0}}\right)-\log \left(\frac{p_{\mathrm{O}_{2}}}{p_{\mathrm{O}_{2}}^{r e f}}\right)\right]-i R_{o h m},
$$

where $E_{r}$ is the ideal potential voltage of the fuel cell, $\alpha$ is the cathode charge transfer coefficient, $R_{o h m}$ is the internal resistance of the membrane and $p_{\mathrm{O}_{2}}$ and $p_{\mathrm{O}_{2}}^{r e f}$ are the oxygen pressure and oxygen pressure reference at the CCL respectively.
TABLE I

PHYSICAL PROPERTIES AND CONSTANTS

\begin{tabular}{|c|c|c|}
\hline Parameter & Description & Units \\
\hline$A$ & Electrode area & $\mathrm{m}^{2}$ \\
\hline$c_{i}$ & Concentration of $i$-th gas & $\mathrm{mol} \mathrm{m}^{-3}$ \\
\hline$D_{i}$ & Diffusion coefficient of $i$-th gas & $\mathrm{m}^{2} \mathrm{~s}^{-1}$ \\
\hline$F$ & Faraday constant & $\mathrm{C} \mathrm{mol}^{-} 1$ \\
\hline$i$ & Current density & $\mathrm{A} \mathrm{m}^{-2}$ \\
\hline$i_{0}$ & Exchange current density & $\mathrm{A} \mathrm{m}^{-2}$ \\
\hline$J$ & Diffusion flux & $\mathrm{mol} \mathrm{m}^{-2} \mathrm{~s}^{-1}$ \\
\hline$L$ & Fuel cell length & $\mathrm{m}$ \\
\hline$L_{x}$ & Gas channel width & $\mathrm{m}$ \\
\hline$L_{z}$ & Gas channel depth & $\mathrm{m}$ \\
\hline$\dot{n}_{i}$ & $\mathrm{y}$-direction flux of $i$-th gas & $\mathrm{mol} \mathrm{m}^{-2} \mathrm{~s}^{-1}$ \\
\hline$n_{y}$ & $y$-direction discretisation volumes & - \\
\hline$n_{z}$ & $z$-direction discretisation volumes & - \\
\hline$p$ & Pressure & $\mathrm{Pa}$ \\
\hline$R$ & Gas constant & $\mathrm{J} \mathrm{mol}^{-1} \mathrm{~K}^{-1}$ \\
\hline$S$ & Water source term & $\mathrm{mol} \mathrm{m}^{-3} \mathrm{~s}^{-1}$ \\
\hline$T$ & Temperature & K \\
\hline$v$ & Flow velocity & $\mathrm{m} \mathrm{s}^{-1}$ \\
\hline$\Delta G_{0}$ & Gibbs activation energy & $\mathrm{J} \mathrm{mol}^{-1}$ \\
\hline$\Delta y$ & $y$-direction discretisation length & $\mathrm{m}$ \\
\hline$\Delta z$ & $z$-direction discretisation length & $\mathrm{m}$ \\
\hline$\delta$ & $y$-axis thickness & $\mathrm{m}$ \\
\hline$\kappa$ & Pressure drop coefficient & $\mathrm{m}^{2} \mathrm{~s}^{-1} \mathrm{~Pa}^{-1}$ \\
\hline
\end{tabular}

The exchange current density $i_{0}$ is a function of the fuel cell temperature, oxygen pressure and the electrochemically active surface area (ECSA) within the CCL $\left(\mathrm{ECSA}_{C C L}\right)$

$$
i_{0}=i_{0}^{r e f} \frac{E C S A_{C C L}}{A_{g e o}}\left(\frac{p_{O_{2}}}{p_{\mathrm{O}_{2}}^{r e f}}\right)^{0.5} e^{\left[-\frac{\Delta G^{*}}{R T}\left(1-\frac{T}{T^{r e f}}\right)\right]},
$$

being $i_{0}^{r e f}$ the intrinsic catalytic Pt activity at normal conditions ( $T^{r e f}$ and $\left.p_{\mathrm{O}_{2}}^{r e f}\right), A_{g e o}$ the total surface area and $\Delta G^{*}$ the Gibbs activation energy for the oxygen reduction reaction.

2) Water model: Water is a key element regarding the performance and efficiency of fuel cells. The amount of liquid water at the CCL directly affects the $\mathrm{ECSA}_{C C L}$ and therefore the voltage of the PEMFC as determined by Eqs. (1) and (2). In this paper, the ratio between the volume of liquid water and the total volume of void space in the porous GDL and CCL is denoted by the liquid water saturation ratio $s$ [2]. The two-phase water model is only implemented in the cathode side of the PEMFC (see Figure 1), disregarding the effect of liquid water at the anode side.

To be able to compute the liquid water saturation ratio in the CCL and GDL, first the general expression for the dynamics of $s$ is developed as introduced in [2]:

$$
\frac{\partial s}{\partial t}=\frac{S_{H_{2} O}^{l}-\nabla \cdot\left(-D_{s} \vec{\nabla} s\right)}{\varepsilon \rho_{H_{2} O}^{l}},
$$

where $D_{s}$ denotes the liquid water diffusivity throughout the layer, $\varepsilon$ the specific porosity, $\rho_{\mathrm{H}_{2} \mathrm{O}}^{l}$ the liquid water density and 
$S_{\mathrm{H}_{2} \mathrm{O}}^{l}$ the liquid water source term for the cathode side of the PEMFC (see first row of Table II). Notice that the expressions for the GDL and CCL are different. $\nabla \cdot \mathbf{F}$ and $\vec{\nabla} f$ denote the mathematical divergence and gradient respectively.

In [2] only the $y$-direction discretisation was considered when developing the divergence and gradient of $s$. In the present paper, the approach will be implemented for the $y$ and $z$-directions, as expressed by

$$
\frac{\partial s}{\partial t}=\frac{S_{H_{2} O}^{l}+D_{s}\left(\frac{\partial^{2} s}{\partial y^{2}}+\frac{\partial^{2} s}{\partial z^{2}}\right)}{\varepsilon \rho_{H_{2} O}^{l}} .
$$

To compute the liquid water source term $S_{\mathrm{H}_{2} \mathrm{O}}^{l}$ at the GDL and CCL, the water generation $\left(S_{H_{2} O}^{g e n}\right)$, water evaporation $\left(S_{H_{2} O}^{\text {evap }}\right)$ and membrane source $\left(S_{H_{2} O}^{m}\right)$ terms are needed. The complete expressions for these terms can be found in [2, 5].

Regarding the membrane source term, it relates the liquid water condition of the CCL with the water content at the membrane $\Lambda$ taking into account the diffusivity from the membrane to the CCL. The water content $\Lambda$ is calculated using the absorption isotherms proposed in [5].

A summary of the source terms for the GDL and CCL is presented in Table II.

3) Gas flow model: The gas species flow dynamics are described by mass balance equations along the PEMFC gas channels (see Figure 1)

$$
\begin{aligned}
\frac{\partial c_{i}}{\partial t} & =\frac{\partial}{\partial z}\left(v c_{i}\right)-\frac{\dot{n}_{i}}{\delta}+S_{i}^{g}, \\
v & =-\kappa \frac{\partial p}{\partial z}, \\
p & =R T \sum_{i} c_{i},
\end{aligned}
$$

being $c_{i}$ the concentration of the $i$-th gas, where subscript $i$ stands for the gaseous species, namely $i=H_{2}$ for the hygrogen, $i=\mathrm{O}_{2}$ for the oxygen, $i=N_{2}$ for the nitrogen and $i=\mathrm{H}_{2} \mathrm{O}$ for the vapour water. The reaction and water molar transports from the membrane electrode assembly (MEA) are modelled in $\dot{n}_{i}$ and they are defined as perpendicular to the gas channels in the y-direction [5]. The $y$-direction thickness of the anode and cathode gas channels is represented by $\delta . S_{i}^{g}$ is a function of the evaporation rate of the liquid water in the cathode side of the PEMFC (see Table II) and is only valid when $i=\mathrm{H}_{2} \mathrm{O}$ at the cathode $\left(S_{i}^{g}=0\right.$ for any other gaseous species and the water at the anode side).

TABLE II

SOURCE TERMS FOR THE CATHODE TWO-PHASE WATER MODEL

\begin{tabular}{lll}
\hline & $\mathrm{GDL}$ & $\mathrm{CCL}$ \\
\hline$\left(\mathrm{H}_{2} \mathrm{O}\right)^{l}$ & $S_{\mathrm{H}_{2} \mathrm{O}}^{l}=-S_{\mathrm{H}_{2} \mathrm{O}}^{\text {evap }}$ & $S_{\mathrm{H}_{2} \mathrm{O}}^{l}=-S_{\mathrm{H}_{2} \mathrm{O}}^{\text {evap }}+S_{\mathrm{H}_{2} \mathrm{O}}^{\text {gen }}+S_{\mathrm{H}_{2} \mathrm{O}}^{m}$ \\
$\left(\mathrm{H}_{2} \mathrm{O}\right)^{g}$ & $S_{\mathrm{H}_{2} \mathrm{O}}^{g}=S_{\mathrm{H}_{2} \mathrm{O}}^{\text {evo }}$ & $S_{\mathrm{H}_{2} \mathrm{O}}^{g}=S_{\mathrm{H}_{2} \mathrm{O}}^{\text {eva }}$ \\
\hline
\end{tabular}

4) Diffusion model: Diffusion is the movement of a substance from a region of high concentration to a region of low concentration. In the PEMFC, the concentration gradient of reactants appears between the gas channels (high concentration) and the CLs (low concentration), where the chemical reactions take place.

Hydrogen, oxygen and water diffuse from the gas channels through the GDLs and CLs in the $y$-direction due to the gradient of concentrations. The diffusion model in the GDLs and CLs of the PEM fuel cell follows Fick's first law of diffusion [7]:

$$
J_{i}=-D_{i} \frac{\partial c_{i}}{\partial y}
$$

being $J_{i}$ and $D_{i}$ the diffusion flux and diffusion coefficient of the $i$-th gas species through the GDLs and CLs respectively, excluding the nitrogen which does not react. The values of the concentrations depend on the position along the $y$-direction. Considering Eq. (6), and the mass continuity equation, the gas concentrations at the boundary CLs after diffusing can be expressed as

$$
\frac{\partial c_{i, C L}}{\partial t}=D_{i} \frac{\partial^{2} c_{i}}{\partial y^{2}}
$$

\section{Finite-difference Discretisation}

The $y$ and $x$ spatial derivatives in the simulation model have to be discretised. In this section, a finite-discretisation procedure is introduced. After being discretised, the model equations are solved numerically with the aid of a computer and a mathematical solver. The model presents several known boundary conditions, such as the input molar fluxes or the external ambient pressure at the end of the gas channels. Henceforth, forward or backward differences will be applied to take advantage from the aforesaid boundary conditions.

The forward difference of a spatial derivative for a generic $x$-dependant function $f(x)$ is expressed as

$$
\frac{\partial f(x)}{\partial x} \approx \frac{f(x+\Delta x)-f(x)}{\Delta x},
$$

while the backward difference follows the expression

$$
\frac{\partial f(x)}{\partial x} \approx \frac{f(x)-f(x-\Delta x)}{\Delta x} .
$$

For the second order spatial differences, the second order central differentiation is expressed as follows:

$$
\frac{\partial^{2} f(x)}{\partial z^{2}} \approx \frac{f(x-\Delta z)-2 f(x)+f(x+\Delta z)}{(\Delta z)^{2}} .
$$

The first order spatial derivatives are solved using Eqs. (8) and (9). Moreover, Eq. (10) is used to solve the second order spatial derivatives.

\section{OBSERVER MODEL}

\section{A. Discretised observer model derivation}

Following the same procedure as with the simulation model, the observation model is discretised following the finitediscretisation procedure presented in Section II-C.

To estimate the amount of active surface in the PEMFC, first, the $\mathrm{ECSA}_{C C L}$ is isolated from Eq. (2)

$$
E C S A_{C C L}=\frac{i_{0} A_{g e o}\left(i_{0, r e f}\right)^{-1}}{e^{\left(-\Delta G^{*}\left(1-\frac{T}{T_{r e f}}\right) R^{-1} T^{-1}\right)}}\left(\frac{p_{O_{2}}}{p_{O_{2}}^{r e f}}\right)^{-0.5}
$$


Assuming that the temperature of the PEMFC is constant and known (as pointed out in the previous section), the only unknowns in Eq. (11) are the current exchange $i_{0}$ and the oxygen partial pressure $p_{\mathrm{O}_{2}}$. The exchange current is obtained from Eq. (1)

$$
i_{0}=i\left(e^{\frac{-R T \log \left(a_{O_{2}}\right)-2 \alpha F V_{f c}+2 E_{r} \alpha F-2 i R_{o h m} \alpha F}{R T}}\right)^{-1}
$$

which leaves the oxygen partial pressure at the CCL as the only unknown parameter. Considering Eq. (5c), the partial oxygen pressure at the CCL can be expressed as

$$
p_{O_{2}, C L}=R T c_{O_{2}, C L}
$$

As introduced previously, the PEMFC is going to be discretised in order to obtain the full concentrations profile. Hence, the partial pressure in Eq. (13) has to be obtained for the full discretisation profile. In this paper, a $n_{z}$ discretisation along the $z$-direction is carried out. Henceforth

$$
p_{O_{2},(j, C L)}=R T c_{O_{2},(j, C L)},
$$

being $j \triangleq\left[1, \ldots, n_{z}\right]$ the discretisation volume subscript.

A Nonlinear Distributed Parameter Observer (NDPO) [4] is implemented to estimate the full profile $(\forall j)$ of all the reactants and water concentrations $(\forall i)$ along the anode and cathode gas channels. Afterwards, applying the diffusion law from Eq. (7) the concentrations at the catalyst site are obtained.

As told, every reactant and water concentrations for all the discretisation volumes are estimated by the observer. However, in this paper, only the model for the oxygen concentrations is presented $\left(i=\mathrm{O}_{2}\right)$ since Eq. (14) requires the amount of oxygen available to react at the catalyst surface. Nevertheless, internally the HOSM observer estimates all the concentration terms for all the reactants [4].

Applying the forward and backward discretisation procedure introduced in Section II-C, the gas flow model in Eq. (5) can be expressed for the oxygen concentration as

$$
\begin{aligned}
\frac{\partial c_{O_{2}, j}}{\partial t} & =\frac{v_{j-1} c_{O_{2}, j-1}}{\Delta z}-\frac{v_{j} c_{O_{2}, j}}{\Delta z}-\frac{\dot{n}_{O_{2}, j}}{\delta}+S_{O_{2}, j}^{g} \\
v_{j} & =\frac{\kappa}{\Delta z}\left(p_{j}-p_{j+1}\right) \\
p_{j} & =R T \sum_{i} c_{O_{2}, j}
\end{aligned}
$$

where $\Delta z$ is the $z$-direction discretisation length, denoted by the division of the number of discretisation volumes by the total length of the gas channel $L_{z}$. Considering the boundary condition for the oxygen given by [5]

$$
\begin{aligned}
v_{i n} c_{O_{2}, i n} & =\dot{n}_{O_{2}, i n} \triangleq u_{O_{2}}, \\
p_{\text {out }} & =p_{n_{z}}=p^{a m b},
\end{aligned}
$$

and a spatial discretisation over $n_{z}$ finite volumes, the oxygen concentration at volume $j$ in Eq. (15) can be expressed as:

$$
\begin{aligned}
& \dot{c}_{O_{2}, j}= \begin{cases}\frac{u_{O_{2}}}{\Delta z}-\zeta_{1} \Psi(j)-\frac{\dot{n}_{O_{2}, j}}{\delta_{C}}, & \text { if } j=1, \\
\zeta_{1} \Psi(j-1)+\Gamma(j+1)-\frac{\dot{n}_{O_{2}, j}}{\delta_{C}}, & \text { if } j=n_{z} \\
\zeta_{1}(\Psi(j-1)-\Psi(j))-\frac{\dot{n}_{O_{2}, j}}{\delta_{C}}, & \text { else, }\end{cases} \\
& y_{O_{2}}=c_{O_{2}, n_{z}},
\end{aligned}
$$

with

$$
\begin{aligned}
\Psi(j) & =c_{O_{2}, j}\left(\sum_{i} c_{i, j}-\sum_{i} c_{i, j+1}\right), \\
\Psi(j-1) & =c_{O_{2}, j-1}\left(\sum_{i} c_{i, j-1}-\sum_{i} c_{i, j}\right), \\
\Gamma(j+1) & =c_{O_{2}, j}\left(\zeta_{1} \sum_{i} c_{i, j}+\zeta_{2}\right),
\end{aligned}
$$

being $\zeta_{1}=\kappa R T / \Delta z^{2}$ and $\zeta_{2}=\kappa p^{a m b} / \Delta z^{2}$.

The concentration of oxygen is then discretised through the GDL and CCL following a spatial discretisation over $n_{y}$, using the diffusion law expressed in Eq. (6) and the second order spatial difference from Eq. (10)

$\dot{c}_{O_{2},(j, k)}=\left\{\begin{array}{l}\frac{D_{O_{2}}}{\Delta y^{2}}\left(c_{O_{2}, j}-2 c_{O_{2},(j, k)}+c_{O_{2},(j, k+1)}\right), \text { if } k=1, \\ \frac{D_{O_{2}}}{\Delta y^{2}}\left(c_{O_{2},(j, k-1)}-2 c_{O_{2},(j, k)}\right), \text { if } k=n_{y}, \\ \frac{D_{O_{2}}}{\Delta y^{2}}\left(c_{O_{2},(j, k-1)}-2 c_{O_{2},(j, k)}+c_{O_{2},(j, k+1)}\right), \text { else }\end{array}\right.$

where $k \triangleq\left[1, \ldots, n_{y}\right]$ is the discretisation volume subscript for the $y$-direction.

In Section IV the observer formulation is given for the estimation of the full oxygen profile that allows to compute Eq. (11) at each $y$ and $z$ discretisation element.

\section{B. Measurements}

As illustrated by Eq. (17b), in order to implement the NDPO and then, the ECSA $C C L$ observer, a measurement of the output oxygen concentration is needed. The complete procedure to estimate the output concentrations from conventional measurements in PEMFC-based systems is explained in [3]. To summarize, to synthesise the ECSA observer, the following measurements are assumed to be available:

- Cathode pressure drop

- Oxygen input molar flux

- Temperatures in the PEMFC

- Demanded current

- PEMFC output voltage

\section{HOSM OBSERVER}

\section{A. Block controllable structure}

To implement the HOSM observer [4], the first step is to express the observer dynamics for the oxygen concentrations described in Eq. (17) following a block controllable structure [6]

$$
\begin{array}{ll}
\dot{\hat{c}}_{O_{2}, n_{z}} & =F_{n_{z}}+B_{n_{z}} \hat{c}_{O_{2}, n_{z}-1}+\hat{G}_{n_{z}}, \\
\dot{\hat{c}}_{O_{2}, n_{z}-1} & =F_{n_{z}-1}+B_{n_{z}-1} \hat{c}_{O_{2}, n_{z}-2}+\hat{G}_{n_{z}-1}, \\
& \vdots \\
\dot{\hat{c}}_{O_{2}, 1} & =F_{1}+B_{1} u+\hat{G}_{1},
\end{array}
$$


where $u$ is the correction input, $F:\left\{n_{z}\right\} \times\{1\} \in \mathbb{R}$ is the vector that includes the nonlinear dynamics that depend on the $j$ and $(j+1)$-discretisation volumes and the input molar flux. Following the notation introduced in Eqs. (17) and (18):

$$
F=\left[\begin{array}{c}
\frac{u_{O_{2}}}{\Delta z}-\zeta_{1} \hat{c}_{O_{2}, 1}\left(\sum_{i} \hat{c}_{i, 1}-\sum_{i} \hat{c}_{i, 2}\right) \\
-\zeta_{1} \hat{c}_{O_{2}, 2}\left(\sum_{i} \hat{c}_{i, 2}-\sum_{i} \hat{c}_{i, 3}\right) \\
\vdots \\
\hat{c}_{O_{2}, n_{z}}\left(\zeta_{1} \sum_{i} \hat{c}_{i, n_{z}}+\zeta_{2}\right)
\end{array}\right] .
$$

$B:\left\{n_{z}\right\} \times\{1\} \in \mathbb{R}$ is the vector that includes the nonlinear dynamics that depend on the $(j-1)$-discretisation volume

$$
B=\left[\begin{array}{c}
1 \\
\zeta_{1}\left(\sum_{i} \hat{c}_{i, 1}-\sum_{i} \hat{c}_{i, 2}\right) \\
\vdots \\
\zeta_{1}\left(\sum_{i} \hat{c}_{i, n_{z}-1}-\sum_{i} \hat{c}_{i, n_{z}}\right)
\end{array}\right] .
$$

And finally, $\hat{G}:\left\{n_{z}\right\} \times\{1\} \in \mathbb{R}$ is the vector of the available rough estimates of the bounded unknown oxygen reaction matrix along the $z$-direction of the PEMFC. Such estimates are introduced in order to further decrease the estimation error on the gas concentration estimates of the fuel cell due to the presence of unmatched uncertainty in the observer error dynamics.

The observation problem is to design a correction input $u$ such that the output observation error $e_{y}=\hat{c}_{O_{2}, n_{z}}-c_{O_{2}, n_{z}}$, is driven in a vicinity of zero with bounded derivatives in a finite amount of time, even with the presence of the unknown bounded perturbations. This procedure will give back the full profile of oxygen concentrations along the CCL.

\section{B. HOSM back-stepping algorithm}

A back-stepping observation algorithm is designed rewriting the control algorithm proposed in [6] for state observation purposes. The observation procedure follows an $r$-step algorithm, where $r \in\left[1,2, \ldots, n_{z}\right]$, as shown in Algorithm 1 .

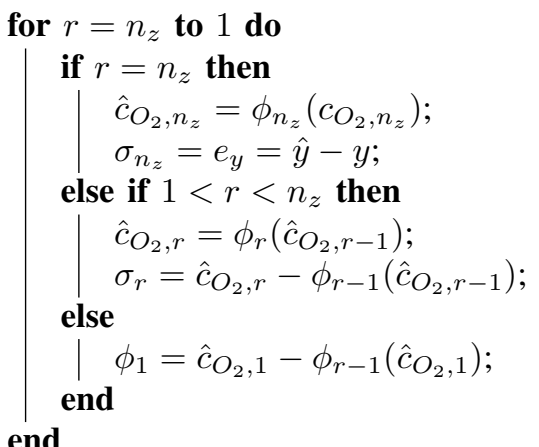

Algorithm 1: HOSM back-stepping algorithm

The quasi-continuous term that has to drive the observation error to zero is included in the $\phi_{r}\left(\hat{c}_{O_{2}, r}\right)$ terms [6], which are defined as

$$
\phi_{r}=D_{r}^{-1}\left\{-F_{r}+u_{r}\right\},
$$

where $D:\left\{n_{z}\right\} \times\left\{n_{z}\right\} \in \mathbb{R}$ is the diagonal matrix

$$
D=\sum_{i}^{n_{z}} E_{i} B e_{i}
$$

being $E:\left\{n_{z}\right\} \times\left\{n_{z}\right\}$ a diagonal matrix with a 1 at each $(i, i)$ position; and similarly $e:\{1\} \times\left\{n_{z}\right\}$ is the row matrix with a 1 on position $(1, i)$ and zeros everywhere else.

The $r$-sliding homogeneous corrective action $u_{r}$ that appears in Eq. (23) has to be designed for $r<n_{z}$

$$
u_{r}=-\alpha_{r} \Psi\left(\sigma, \dot{\sigma}, \ldots, \sigma^{r-1}\right) \text {, }
$$

with $\sigma_{1}=\hat{c}_{O_{2}, 1}-\phi\left(\hat{c}_{O_{2}, 1}\right)$. The computation of the derivatives $\sigma, \dot{\sigma}, \ldots, \sigma^{r-1}$ in Eq. (25) is made using robust differentiators with finite-time convergence [8].

Once the concentrations estimation along the gas channels is achieved, the diffusion concentrations observation for Eq. (19) is carried out using an observer with similar structure as in Eq. (20) (not included due to space limitations).

\section{Convergence of the Observer}

The HOSM observer has a relative degree equal to $n_{z}$. As shown in Algorithm 1, the correction term for $1 \leq r<n_{z}$ is obtained from the observed states $\hat{c}_{O_{2}, j}$. This introduces an accumulated error when applying the back-stepping algorithm. As it will be shown in the following section, this error can be considered negligible and the observer performs well under a given simulation scenario. Nevertheless, improvements towards reducing the accumulated error and analysing the convergence of the observer are due in future research.

\section{Simulations}

\section{A. Numerical implementation}

The mesh for the simulation and observation models consists of 5 elements equally distributed along the $z$-direction, 5 elements for the GDL and 5 for the CCL along the $y$ direction. The observer for the $\operatorname{ECSA}_{C C L}$ is initialised with

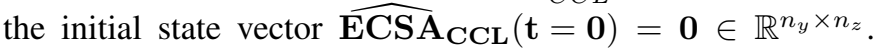
Regarding the oxygen concentrations observer $\hat{\mathbf{c}}_{\mathrm{O}_{2}}(\mathbf{t}=\mathbf{0})=$ $\mathbf{0} \in \mathbb{R}^{1 \times n_{z}}$. Finally, since a full state observer is used, the initial conditions for the remainder $i$-th gas concentrations observer are $\hat{\mathbf{c}}_{\mathbf{i}}(\mathbf{t}=\mathbf{0})=\mathbf{0} \in \mathbb{R}^{4 \times n_{z}}$. Simulations have been carried out using MATLAB R2011a (32 bits), running in a PC Intel Core i7-3770 at $3.40 \mathrm{GHz}$ with $8 \mathrm{~GB}$ of RAM

\section{B. Case study}

To test the dynamic performance of the developed observation strategy, a New European Driving Cycle (NEDC) current density profile [9] is going to be applied to the simulation model. The employed NEDC current density profile is shown in the right axis of Figure 2. The full simulation model introduced in Section II is used as the plant while the observer model follows the dynamics presented in Eq. (20). The PEMFC is operated with anode and cathode stoichiometric rates of 1.3 and 2.0 respectively, a constant inlet pressure of 1.23 bar, a PEMFC temperature of $353 \mathrm{~K}$ and a constant humidity of $50 \%$ for both gas channel inputs. 


\section{Results and discussion}

The comparison between the modelled ECSA $_{C C L}$ and the observed variable $\widehat{E C S A_{C C L}}$ for different simulation conditions is shown in Figure 2. These conditions include the observation under different evaporation constant $\left(K_{\text {evap }} \in\right.$ $\{500 ; 1000\})$ values for the liquid water content in the CCL $\left(S_{H_{2} O, C C L}^{l}=f\left(K_{\text {evap }}\right)\right)$, a variable that is unknown from the point of view of the observation model.

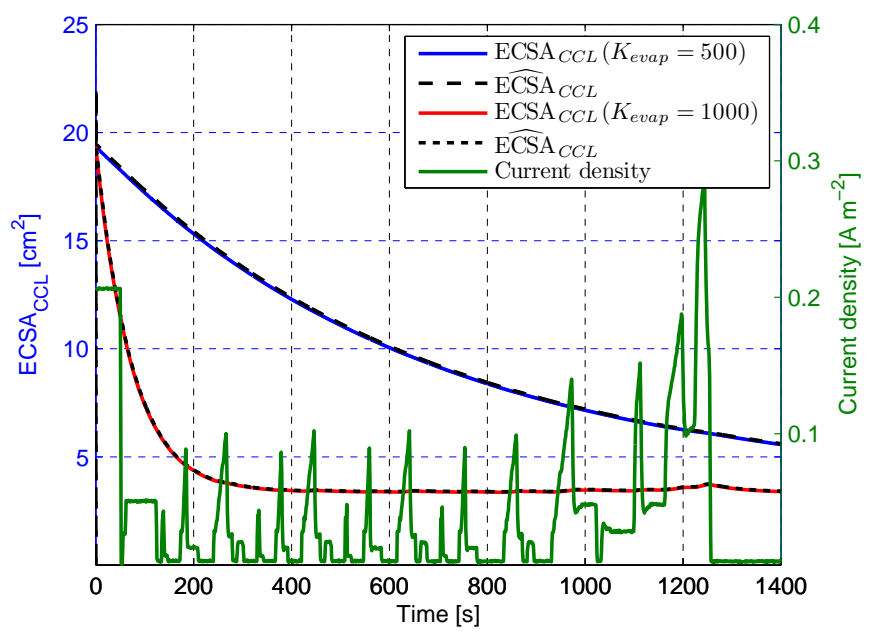

Fig. 2. Observation of $\mathrm{ECSA}_{C C L}$

The variable $\mathrm{ECSA}_{C C L}$ in Figures 2 and 3 represents the total active surface in the CCL $\left(\mathrm{ECSA}_{C C L}=\sum_{j, k} \mathrm{ECSA}_{j, k}\right.$, $\forall(j, k))$. Nevertheless, the computation is done for each one of the $n_{y} \times n_{z}$ CCL discretisation elements. The observation is performed properly throughout the simulation time. The detail of the convergence is shown in Figure 3. As expected, the amount of active surface depends on the ratio of liquid water present in the CCL, which varies depending on the evaporation rate value. The demanded current also affects the amount of generated water as it can be seen in Figure 2 around $1200 \mathrm{~s}$.

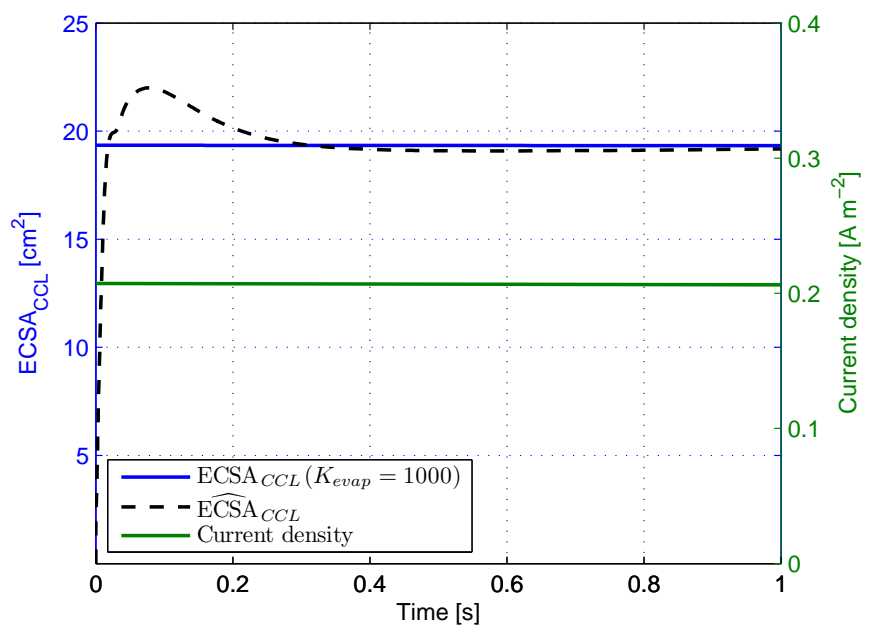

Fig. 3. Observation of ECSA $_{C C L}$ (detail)

\section{CONCLUSION}

In this paper, a methodology to observe the ECSA in a PEMFC catalyst layer is proposed and tested under simulation for a NEDC current profile demand. The observer is able to estimate the active surface in the PEMFC CCL, a magnitude that is directly related to the liquid water present in the system. The performance of the strategy has been evaluated, obtaining satisfactory results for the proposed simulation scenario.

The methodology developed is suitable to be implemented in control strategies in order to use the humidification of the PEMFC to improve the ECSA value and increase the efficiency of the reaction at the CCL. Moreover, the observer is discretised along the $z$ and $y$-directions, giving the possibility of implementing distributed parameters control solutions that take into account the inner conditions of the fuel cell which is critical for the improvement of performance and durability.

\section{ACKNOWLEDGEMENT}

This work has been partially funded by the MINECO/FEDER project DPI2015-69286-C3-2-R, the Regione Autonoma della Sardegna project CRP-7733 and the European project PUMA-MIND (ref. SPI-JTI-FCH 303419).

\section{REFERENCES}

[1] F. Barbir, PEM fuel cells: theory and practice. Elsevier Academic Press, 2013.

[2] S. Strahl, A. Husar, and A. A. Franco, "Electrode structure effects on the performance of open-cathode proton exchange membrane fuel cells: A multiscale modeling approach," International Journal of Hydrogen Energy, vol. 39, no. 18, pp. 9752-9767, 2014.

[3] J. Luna, A. Husar, and M. Serra, "Nonlinear distributed parameter observer design for fuel cell systems," International Journal of Hydrogen Energy, vol. 40, pp. 11322$11332,2015$.

[4] J. Luna, E. Usai, A. Husar, and M. Serra, "Nonlinear observation in fuel cell systems: A comparison between disturbance estimation and high-order sliding-mode techniques," International Journal of Hydrogen Energy, 2016.

[5] M. Mangold, A. Bück, and R. Hanke-Rauschenbach, "Passivity based control of a distributed PEM fuel cell model," Journal of Process Control, vol. 20, no. 3, pp. 292-313, 2010.

[6] A. Estrada and L. Fridman, "Quasi-continuous HOSM control for systems with unmatched perturbations," $\mathrm{Au}$ tomatica, vol. 46, no. 11, pp. 1916-1919, 2010.

[7] R. Taylor and R. Krishna, Multicomponent mass transfer. John Wiley \& Sons, 1993, vol. 2.

[8] A. Levant, "Higher-order sliding modes, differentiation and output-feedback control," International Journal of Control, vol. 76, no. 9-10, pp. 924-941, 2003.

[9] M. Mayur, S. Strahl, A. Husar, and W. G. Bessler, "A multi-timescale modeling methodology for pemfc performance and durability in a virtual fuel cell car," International Journal of Hydrogen Energy, vol. 40, no. 46, pp. 16 466-16476, 2015. 